\title{
Selective FGFR inhibitor BGJ398 inhibits phosphorylation of AKT and STAT3 and induces cytotoxicity in sphere-cultured ovarian cancer cells
}

\author{
HWA JUN CHA ${ }^{1}$, JUNG HYE CHOI ${ }^{2}$, IN CHUL PARK ${ }^{3}$, CHUN HO KIM $^{4}$, \\ SUNG KWAN AN ${ }^{5}$, TAE JIN KIM ${ }^{6,7}$ and JAE HO LEE ${ }^{7}$
}

\begin{abstract}
${ }^{1}$ Osan University, Department of Skin Care and Beauty, Osan, Gyeonggi-do 18119; ${ }^{2}$ Department of Oriental Pharmacy, College of Pharmacy, Kyung Hee University, Seoul 02447; ${ }^{3}$ Division of Radiation Cancer Research, Korea Institute of Radiological and Medical Sciences, Seoul 01812; ${ }^{4}$ Laboratory of Tissue Engineering, Korea Institute of Radiological and Medical Science, Seoul 139240; ${ }^{5}$ Molecular-Targeted Drug Research Center, Konkuk University, Seoul 05029; ${ }^{6}$ Department of Obstetrics and Gynecology, Cheil General Hospital and Women's Healthcare Center, Dankook University College of Medicine, Seoul 04619; ${ }^{7}$ Laboratory of Molecular Oncology, Cheil General Hospital and Women's Healthcare Center, Dankook University College of Medicine, Seoul 04619, Republic of Korea
\end{abstract}

Received December 3, 2016; Accepted March 3, 2017

DOI: 10.3892/ijo.2017.3913

\begin{abstract}
Epithelial ovarian cancer is the most aggressive and lethal among the gynecological malignancies, which is often found disseminated to peritoneal cavity at the time of diagnosis. There is accumulating evidence on the existence of genetic alteration and amplification of fibroblast growth factor receptor (FGFR) in various cancers. Also the aberrated FGFR/FGF signaling has been implicated in cancer development and tumor microenvironment. However, the antitumor activity of BGJ398, a selective inhibitor of FGFR 1/2/3 against ovarian cancer still remains unknown. The aim of the present study is to evaluate the antitumoral activity of BGJ398 on ovarian cancer cell line SKOV3ip1 using 3-dimensional (3D) sphere culture system which has been accepted as a better mimic in vivo microenvironment than conventional 2-dimensional (2D) monolayer culture system. We examined the differential expression features of key signaling molecules which have a role in cell survival and proliferation between sphere-cultured SKOV3ip1 cells and monolayer-cultured SKOV3ip1 cells. The phosphorylation of AKT and signal transducer and activator of transcription 3 (STAT3) known as survival signaling molecules were upregulated in spherecultured SKOV3ip1 cells compared to in monolayer-cultured SKOV3ip1 cells. Next, we evaluated the antitumor activity
\end{abstract}

Correspondence to: Professor Jae Ho Lee, Laboratory of Molecular Oncology, Cheil General Hospital and Women's Healthcare Center, Dankook University College of Medicine, 17 Seoae-ro 1-gil, Jung-gu, Seoul 04619, Republic of Korea

E-mail: jaeho3909@hanmail.net

Key words: BGJ398, ovarian cancer, sphere culture, AKT, STAT3, FGFR of BGJ398 in monolayer-cultured SKOV3ip1 cells or spherecultured SKOV3ip1 cells. Treatment of BGJ398 did not affect the SKOV3ip1 cell viability in monolayer culture system, but, the cell viability of sphere-cultured SKOV3ip1 cells was markedly reduced by BGJ398. The phosphorylation of AKT and STAT3 was downregulated by BGJ398 in sphere-cultured SKOV3ip1 cells, but not in monolayer cultured-SKOV3ip1 cells. Moreover, combination treatment with BGJ398 and paclitaxel in sphere-cultured SKOV3ip1 showed synergistic inhibitory effect on cell viability. Collectively, our report reveals the BGJ398 is a potent antitumor agent against ovarian cancer and FGFR is a promising therapeutic target to anticancer therapy considering ovarian cancer metastatic microenvironment.

\section{Introduction}

Epithelial ovarian carcinomas (EOCs) account for $90 \%$ of total cases of ovarian cancer. Unfortunately, almost $70 \%$ of women with ovarian cancer are not diagnosed until the disease is advanced in stage leading to the highest mortality of any of the gynecologic cancers (1). Paclitaxel has been used as a first-line chemotherapeutic agent against ovarian cancer (2). However, the development of chemoresistance causes the primary failure in the treatment of ovarian cancer (3). Although improvement in survival rate has been observed, the majority of patients experience recurrent disease due to resistance to chemotherapy (4). The identification of suitable biomarkers for chemosensitivity diagnosis to paclitaxel and the development of new therapeutic agent for overcoming chemoresistance may be important research fields to improve the therapeutic outcome of patient with ovarian cancer.

The fibroblast growth factor receptors (FGFRs) consist of an extracellular ligand domain with three immunoglobulinlike domains (I-III), a transmembrane domain, and an 
intracellular tyrosine kinase domain that transmit the signal to the intracellular binding proteins (5). There are four FGFRs (FGFR1/2/3/4) as membrane-bound receptors with seven isoforms due to the alternative splicing in Ig-III-like domain with different ligand-binding affinity (5). While each FGFR can be activated by several FGFs, the FGF/FGFR signaling is further controlled with different tissue distribution $(5,6)$. For example, high level expression of FGFR1 was observed in the skin, cornea, lung, heart, placenta, kidney and uterus. In contrast, high expression of FGFR2 was detected in prostate and stomach. The expressions of FGFR3 and FGFR4 were more restricted than FGFR1 and FGFR2. FGFR3 expression is found in appendix, colon, liver, sublingual gland, placenta, and cervix with restricted and lower patterns. FGFR4 expression is observed in the liver, sublingual gland duct, kidney and ureter (6). Differential tissue distribution of FGFR expressions suggests that FGFR families play important functional roles in normal tissue homeostasis (7). Recent studies have indicated that FGFR signaling is also implicated in tumor development (8-10). Cumulative evidence revealed that FGFR aberrations are common in a wide variety of cancers, with the majority being gene amplification or activating mutations (11). Helsten et al (12) reported that FGFR aberrations were found in $7.1 \%$ of cancers using next-generation sequencing. Moreover, ovarian cancer is the fifth mosy commonly altered ( $9 \%)$ in FGFR activity among the cancer malignancies implicating that FGFR inhibition could be an important therapeutic approach against ovarian carcinoma (12).

$\mathrm{AKT} / \mathrm{PKB}$ is an intracellular serine/threonine kinase. It has been extensively studied that AKT regulates a variety of cellular processes by mediating extracellular and intracellular signals (13-16). Activation of AKT mediates its pleckstrin homology $(\mathrm{PH})$ domain binding to products of phosphatidylinositol 3-kinase (PI3K). This activation is feedback regulated by a dual phosphatase PTEN (17). Activation of AKT through platelet-derived growth factor (PDGF), insulin, epidermal growth factor (EGF), basic fibroblast growth factor (bFGF) has been identified (18-21). These events regulate cell growth, survival, differentiation, angiogenesis, migration and metabolism (22). Moreover, studies have shown that AKT signaling is frequently impaired in many malignancies (23) and the overexpression of AKT induces chemoresistance (24). To date, many studies have demonstrated that AKT signaling is the major target for anticancer drug development and overcoming chemoresistance.

Three-dimensional sphere culture model, a strategy with cell anchorage-independent growth potential, have been reported to establish reliable methodologies and techniques for the high-throughput drug development against various cancers (25-27). Although the sphere culture model is a better recapitulating system of primary tumors than conventional monolayer culture model, the sphere culture model approaches have not yet been used widely in cancer research and drug development fields because of higher costs and technical problems than conventional monolayer culture model (28). The wide application of sphere culture model in cancer cell research and the accumulation of available data through the sphere culture model may facilitate the understanding of tumor growth and metastasis in specific tumor microenvironment $(29,30)$. Especially, epithelial ovarian cancer frequently spreads by direct metastasis from the primary site (31). Unlike primary tumor in ovary, premetastatic ovarian cancer cells undergo epithelial-to-mesenchymal (EMT) transition, which loosens the intercellular attachment between cancer cell-to-cell and cancer cell-to-extracellular matrix through the remodeling of cadherins (31). Detached ovarian cancer cells from the primary site disseminate within the abdominal cavity and often associated with ascitic fluid, particularly in high-grade serous carcinoma. In addition, the disseminated ovarian cancer is faced with a harsh growth condition with the specific tumor microenvironment involving hypoxia and nutrient-deprived conditions (31). Nonetheless, to date the conventional monolayer ovarian cancer culture model has been used for ovarian cancer research preferentially. Here, we report the molecular characterization of ovarian carcinoma SKOV3ip1 cell line in conventional monolayer culture model and sphere cultured model. We evaluated the antitumor activity of the selective FGFR inhibitor BGJ398 against SKOV3ip1 in sphere culture model. These data suggest that BGJ398 is a potent anticancer drug candidate against epithelial ovarian carcinoma. The present data also showed that the inhibition of FGFR/AKT signaling pathway represents a therapeutic target for overcoming metastatic ovarian carcinoma.

\section{Materials and methods}

Antibodies and reagents. Antibodies against STAT3 (H-190), phosphorylated STAT3 at Tyr 705 (B-7) and Actin (C-2) were purchased from Santa Cruz Biotechnology (Santa Cruz, CA, USA). Rabbit monoclonal antibodies against AKT, phosphorylated AKT at Ser473 (D9E), phosphorylated FGFR1 at Tyr 653/654, p42/44 MAPK, phosphorylated p42/44 MAPK, p38 MAPK and phosphorylated p38 MAPK at Tyr 180/182 antibodies were from Cell Signaling Technology (Danvers, MA, USA). Goat anti-mouse and rabbit secondary antibodies conjugated to horseradish peroxidase (HRP) were purchased from Jackson Laboratories (Bar Harbor, ME, USA). BGJ398 (against FGFR1/2/3), TAE684 (against ALK) and imatinib (against Abl) were purchased from Selleck Chemicals LLC (Houston, TX, USA). AG490 (against JAK) was from Tocris Bioscience (Bristol, UK). All reagents were solubilized with dimethyl sulfoxide (DMSO) at the following concentration, paclitaxel at $100 \mu \mathrm{M}$, BGJ398 at $5 \mathrm{mM}$, TAE684 at 3 and imatinib at $5 \mathrm{mM}$.

Cell lines and sphere culture. SKOV3ip1 ovarian carcinoma cell line (32) was a kind gift from Professor A.K. Sood (University of Texas MD Anderson Cancer Center, Houston, TX, USA). Cells were maintained as a $2 \mathrm{D}$ monolayer in RPMI-1640 medium (Corning Incorporated, Corning, NY, USA) supplemented with $10 \%$ fetal bovine serum (FBS; Gibco, Grand Island, NY, USA) and $10 \mathrm{U} / \mathrm{ml}$ penicillin/streptomycin (Gibco) at $37^{\circ} \mathrm{C}$ in a $5 \% \mathrm{CO}_{2}$ humidified incubator. To culture as $3 \mathrm{D}$ sphere system, SKOV3ip1 cells $\left(1 \times 10^{6}\right)$ were seeded in ultra-low attachment 6-well plate (Corning Incorporated) and were cultured in RPMI-1640 medium supplemented with $10 \%$ FBS (Gibco) and $10 \mathrm{U} / \mathrm{ml}$ penicillin/streptomycin (Gibco) at $5 \% \mathrm{CO}_{2}$ incubator.

Immunoblotting. The expression of protein and their phosphorylation status in SKOV3ip1 cells was detected by 
immunoblotting. Monolayer-cultured or sphere-cultured SKOV3ip1 cells were harvested and washed once with icecold $1 \mathrm{X}$ phosphate-buffered saline (PBS), and then lysed by $100 \mu \mathrm{l}$ ice-cold RIPA buffer (20 mM Tris-Cl, pH 8.0, $125 \mathrm{mM}$ $\mathrm{NaCl}, 100 \mathrm{mM}$ phenylmethylsulfonyl fluoride, $1 \mathrm{mM}$ ethylene diamine tetraacetic acid, $1 \%$ Triton $\mathrm{X}-100,0.5 \%$ sodium deoxycholate, $0.1 \%$ sodium dodecyl sulfate, $1 \mathrm{X}$ Complete Protease Inhibitor Cocktail) (Roche, Mannheim, Germany) on ice. Protein concentrations were determined using Bradford protein assay kit (Bio-Rad Laboratories, Hercules, CA, USA). Equal amount of proteins $(30 \mu \mathrm{g})$ were separated by SDS-PAGE and transferred to nitrocellulose membranes, followed by immunoblotting with the specific antibodies and horseradish peroxidase-conjugated secondary antibodies. Immunoreactive bands were detected by SuperSignal West Pico Chemiluminescent Substrate (Thermo Fisher Scientific, Rockford, IL, USA) using Fusion Solo chemiluminescence analyzer (Vilber Lourmat, Marne la Vallee, France).

Cell viability assay. The crystal violet staining assay was examined to determine the cell viability in monolayer culture and sphere culture model. To examined the crystal violet staining assay in monolayer culture model, SKOV3ip1 cells $\left(5 \times 10^{4}\right)$ were seeded in conventional 24 -well plate and incubated overnight. The drugs were then treated with the following concentration to each well; vehicle control (DMSO); paclitaxel at $3.125,6.25,12.5,25,50$ and $100 \mathrm{nM}, \mathrm{BGJ} 398$ at $0.3125,0.625,1.25,2.5$ and $5 \mu \mathrm{M}$, TAE684 at $0.1875,0.375$, $0.75,1.5$ and $3 \mu \mathrm{M}$, and imatinib at $0.3125,0.625,1.25,2.5$ and $5 \mu \mathrm{M}$. Cells were further incubated for $72 \mathrm{~h}$. Three hundred microliter $0.2 \%$ crystal violet solution was added to each well and further incubated for $20 \mathrm{~min}$ with gently shaking. Stained cells were washed with distilled water until a clear background was visible. For colorimetric analysis, crystal violet dye in each well was extracted using $1 \%$ SDS/PBS solution and the the absorbance was determined using EMax PLUS microplate reader (Molecular Devices, Sunnyvale, CA, USA) at $570 \mathrm{~nm}$ wavelength. For determination of cell viability in sphere-cultured cells, SKOV3ip1 cells $\left(5 \times 10^{4}\right)$ were seeded in ultra-low attachment 24-well plate and incubated overnight. Then drugs were added and the cells were further incubated for $72 \mathrm{~h}$. Cells in each well were harvested and transferred to conventional 24-well plate and further incubated for $12 \mathrm{~h}$. Attached viable cells were stained by $0.2 \%$ crystal violet solution for 20 min with gently shaking. Stained cells were washed with distilled water. For colorimetric analysis, crystal violet dye was extracted using $1 \%$ SDS/PBS then the absorbance was determined at $570 \mathrm{~nm}$ using EMax PLUS microplate reader (Molecular Devices).

MTS assay. The cell viability was analyzed by MTS assay in monolayer culture and sphere culture model. For the MTS assay, EZ-Cytox (Daeillab Service, Seoul, Korea) was used following the manufacturer's instructions. SKOV3ip1 cells were seeded into conventional 96-well plate or ultra-low attachment 96 -well plate at a density of $1 \times 10^{4} /$ well and incubated overnight. The drugs were then treated with the following concentration to each well; vehicle control (DMSO); paclitaxel at $3.125,6.25,12.5,25,50$ and $100 \mathrm{nM}, \mathrm{BGJ} 398$ at 0.3125 , $0.625,1.25,2.5$ and $5 \mu \mathrm{M}$, TAE684 at $0.1875,0.375,0.75,1.5$
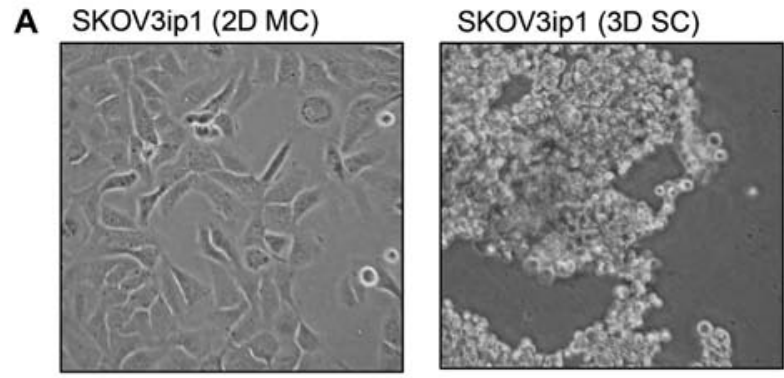

B $\mathrm{MC} \mathrm{SC}$

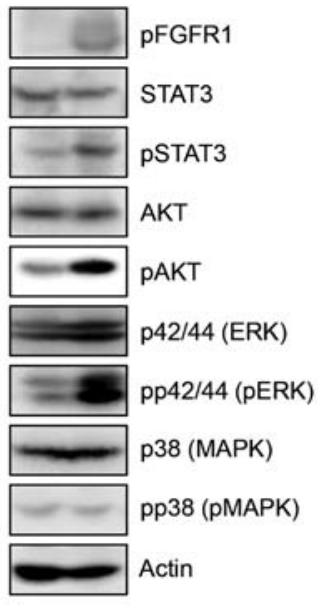

Figure 1. Differential expression pattern of signal molecules in cell survival between monolayer-cultured and sphere-cultured SKOV3ip1 ovarian cancer cells. (A) Monolayer-cultured and sphere-cultured SKOV3ip1 cells were visualized using microscopy. (B) SKOV3ip1 cells $\left(1 \times 10^{6}\right)$ were seeded in conventional plate or ultra-low attached plate and further incubated for $72 \mathrm{~h}$. Cell lysates from monolayer-cultured (2D MC) SKOV3ip1 cells and spherecultured (3D SC) SKOV3ip1 cells were subjected to immunoblotting to analyze the expression level of AKT, STAT3, pp42/44 ERK and p38 MAPK and its activation status. The phosphorylated FGFR1 at Tyr 653/654 residues was also examined.

and $3 \mu \mathrm{M}$, and imatinib at $0.3125,0.625,1.25,2.5$ and $5 \mu \mathrm{M}$. Cells were further incubated for $72 \mathrm{~h}$. Sphere-cultured cells in ultra-low attachment 96-well plate were harvested and transferred to conventional 96-well plate and further incubated for $12 \mathrm{~h}$. Wells with culture media were used as negative control. The absorbance was determined at $450 \mathrm{~nm}$ using EMax PLUS microplate reader (Molecular Devices).

Statistical analysis. All statistical analyses of data were performed from three independent experiments. All experiments were repeated three times. Significant differences by concentration were calculated using one-way ANOVA test and $\mathrm{P} \leq 0.05$ analyzed by concentration were considered significant. All graphs showed the mean with the standard deviation. Cell viability data were analyzed with GraphPad Prism 6 statistical software and presented as the mean value of viable cells \pm standard deviation (SD).

\section{Results}

Differential expressions of survival signaling molecules in SKOV3ipl ovarian cancer cell cultured in conventional $2 D$ monolayer culture model and 3D sphere culture model. We examined the morphological analysis of SKOV3ip1 ovarian 
A Crystal violet colorimetric assay
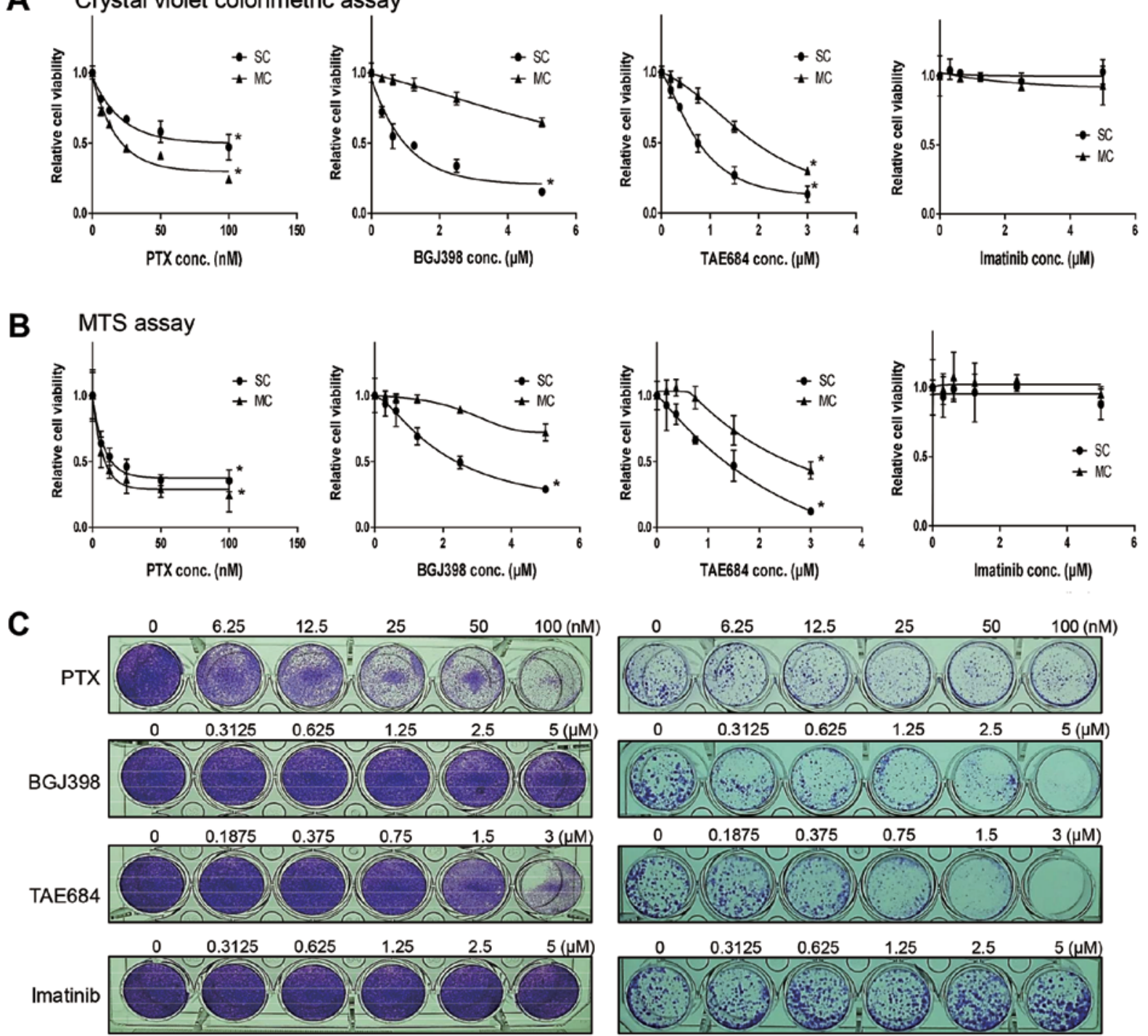

SKOV3ip1 (2D MC)

SKOV3ip1 (3D SC)
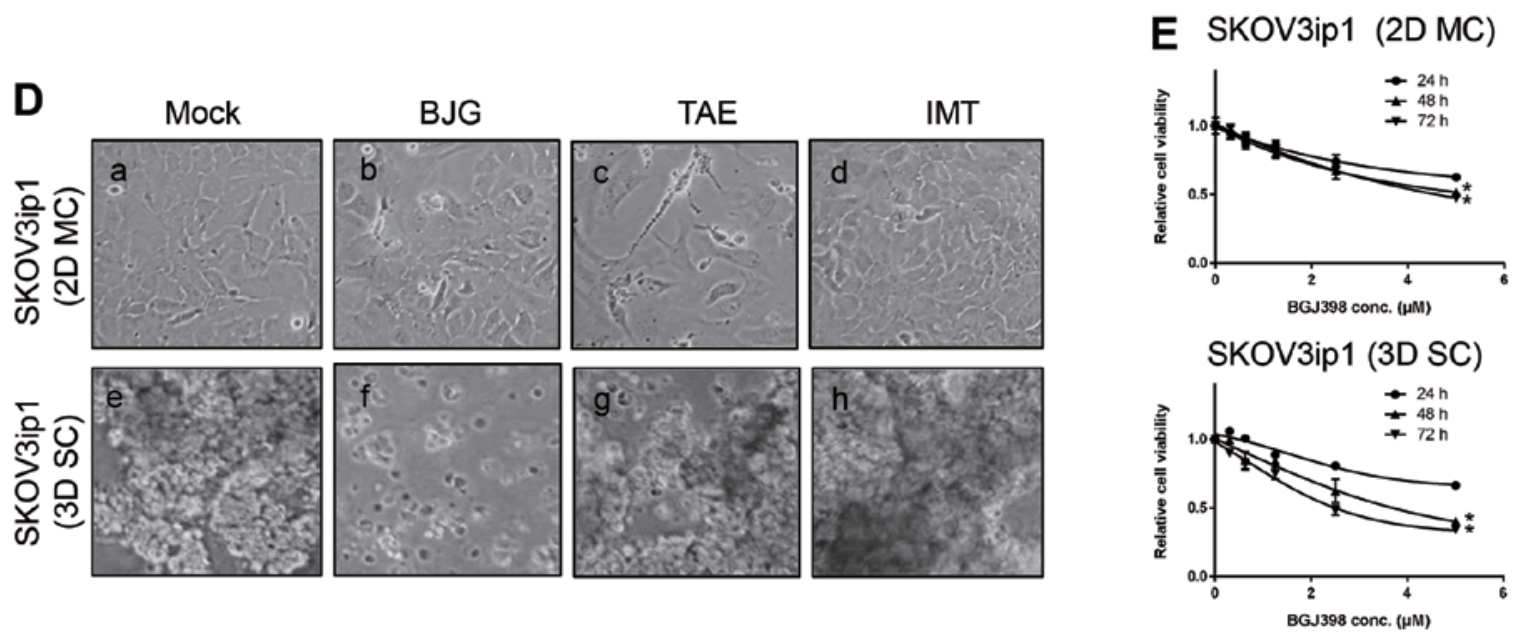

Figure 2. Selective FGFR inhibitor BGJ398 reduces the cell viability in sphere-cultured SKOV3ip1. Cells $\left(5 \times 10^{4}\right)$ were seeded in conventional 24 -well plate and incubated overnight. Then drugs were added with the following concentration to each well; vehicle control DMSO (mock); paclitaxel at 3.125, 6.25, 12.5, 25, 50 and $100 \mathrm{nM}, \mathrm{BGJ} 398$ at $0.3125,0.625,1.25,2.5$ and $5 \mu \mathrm{M}$, TAE684 at $0.1875,0.375,0.75,1.5$ and $3 \mu \mathrm{M}$, and imatinib at $0.3125,0.625,1.25,2.5$ and $5 \mu \mathrm{M}$. Cells were further cultured for $72 \mathrm{~h}$ and the cell viability was determined by crystal violet colorimetric analysis (A) and MTS assay (B). (C) Viable cells at $72 \mathrm{~h}$ after treatment paclitaxel or inhibitors as indicated concentration were visualized by crystal violet staining. (D) Monolayer (2D MC) or spheroid formation (3D SC) of SKOV3ip1 at $72 \mathrm{~h}$ after treatment of vehicle solution DMSO (Mock), $5 \mu \mathrm{M}$ BGJ398 (BGJ), $3 \mu \mathrm{M}$ TAE648 (TAE) and $5 \mu \mathrm{M}$ imatinib (IMT). (E) SKOV3ip1 cells $\left(5 \times 10^{4}\right)$ were seeded in conventional 24 -well plate and incubated overnight. Then DMSO (mock) or BGJ398 $(0.3125,0.625,1.25,2.5$ and $5 \mu \mathrm{M})$ were added to each well. The cell viability was determined by crystal violet colorimetric analysis at 24,48 and $72 \mathrm{~h}$. All experiments were repeated three times. Significant differences by concentration were calculated using one-way ANOVA test and " $\mathrm{P} \leq 0.05$ analyzed by concentration were considered significant. 
A

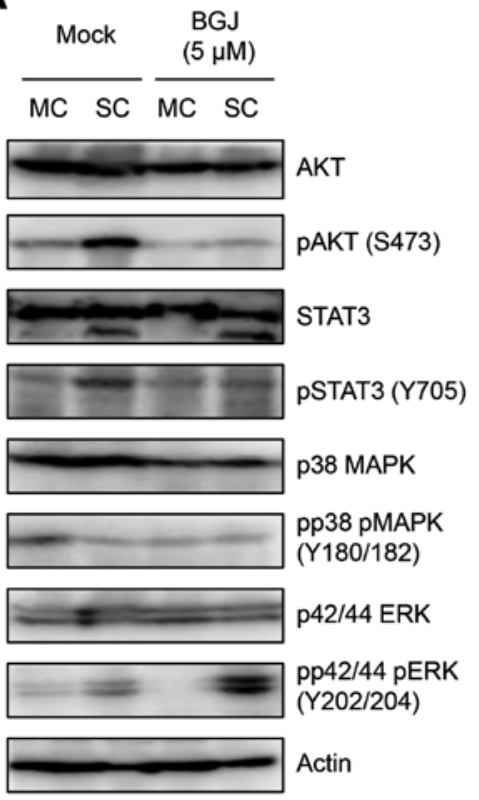

B

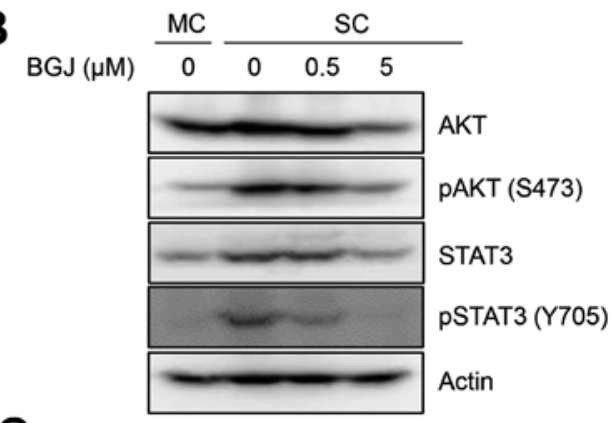

C

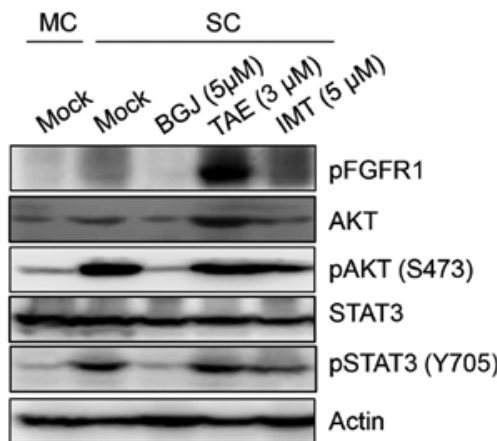

Figure 3. BGJ398 suppresses the phosphoryaltion of AKT and STAT3 in sphere-cultured SKOV3ip1. (A) Monolayer-cultured (MC) and sphere-cultured (SC) SKOV3ip1 cells were treated with DMSO (Mock) and $5 \mu \mathrm{M}$ BGJ398, respectively. At $72 \mathrm{~h}$ after treatment, cells were harvested and were analyzed the activation status of AKT, STAT3, p38 MAPK and p42/44 ERK by immunoblotting. (B) SKOV3ip1 cells were treated with DMSO (Mock) and 0.5 and $5 \mu$ M BGJ398, respectively. At $72 \mathrm{~h}$ after treatment, immunoblotting was performed to analyze the cellular expression and activation of AKT and STAT3 with indicated antibodies. (C) Cell lysates at $72 \mathrm{~h}$ after treatment of DMSO (Mock), $5 \mu \mathrm{M}$ BGJ398 (BGJ), $3 \mu \mathrm{M}$ TAE648 (TAE), and $5 \mu \mathrm{M}$ imatinib (IMT) in sphere-cultured SKOV3ip1 were subjected to immunoblotting and the cellular expression of AKT, STAT3 and its activation status were analyzed with indicated antibodies. The level of phosphorylated FGFR1 was shown as a positive control of BGJ treatment. Actin was used as a loading control.

cancer cells in conventional $2 \mathrm{D}$ monolayer culture and $3 \mathrm{D}$ sphere culture model. At $72 \mathrm{~h}$ after sphere cultivation, SKOV3ip1 cells formed loose sheet-like aggregates and did not accumulate as compact spheroids (Fig. 1A). We examined the expression of cellular signaling molecules which have a role in cell proliferation including AKT, STAT3, p42/44 ERK and p38 MAPK and their activation status in monolayercultured SKOV3ip1 cells and sphere-cultured SKOV3ip1 cells. Immunoblotting showed that the expression level of molecules was not different in monolayer-cultured SKOV3ip1 cells and sphere-cultured SKOV3ip1 cells. Notably, p42/44 ERK, AKT and STAT3 were activated in sphere-cultured SKOV3ip1 cells than in 2D monolayer-cultured SKOV3ip1 cells (Fig. 1B). Additionally, phosphorylated FGFR1 was upregulated in sphere-cultured SKOV3ip1 cells (Fig. 1B). These results showed that the sphere culture condition upregulated the prosurvival signaling pathway in SKOV3ip1 cells.

Selective pan-FGFR inhibitor BGJ398 inhibits cell viability of SKOV3ipl cells in sphere culture model. FGFR inhibitor has been reported as a novel agent with potential anti-angiogenic and anticancer effect (33). It has been reported that FGFR signaling could regulate PI3K/AKT and STAT3 pathway in mammalian cells (34). Thus, we examined the effect of a highly selective pan-FGFR inhibitor BGJ398 on SKOV3ip1 cell viability. Tyrosine kinase inhibitor TAE684 (against ALK) and imatinib also known as Gleevec (against ABL), and standard anti-ovarian cancer chemotherapeutic agent paclitaxel were used and compared in antitumor activities of SKOV3ip1 cells. Monolayer-cultured SKOV3ip1 cells were treated with various concentrations [vehicle control (DMSO)]; paclitaxel at $3.125,6.25,12.5,25,50$ and $100 \mathrm{nM}, \mathrm{BGJ} 398$ at 0.3125 , $0.625,1.25,2.5$ and $5 \mu \mathrm{M}$, TAE684 at $0.1875,0.375,0.75,1.5$ and $3 \mu \mathrm{M}$, and imatinib at $0.3125,0.625,1.25,2.5$ and $5 \mu \mathrm{M}$ ) of tyrosine kinase inhibitors and paclitaxel for $72 \mathrm{~h}$, and the cell viability was determined using crystal violet colorimetric analysis (Fig. 2A) and MTS assay (Fig. 2B). Visualization of crystal violet stained cells confirmed the result of colorimetric analysis (Fig. 2C). As shown in Fig. 2A and B, monolayercultured SKOV3ip1 cell viability was effectively decreased by paclitaxel and TAE648 in a dose-dependent manner $(\mathrm{P}>0.05)$. But the cell viability of monolayer-cultured SKOV3ip1 was not affected by BGJ398 and imatinib (Fig. 2A). Next, the cell viability of sphere-cultured SKOV3ip1 by treatment of paclitaxel or tyrosine kinase inhibitors was compared. Cell viability analysis showed that sphere-cultured SKOV3ip1 cells were clearly more resistant to paclitaxel than SKOV3ip1 in monolayer (Fig. 2A). TAE648-resistance was not observed in sphere-cultured SKOV3ip1 cells (Fig. 2A). Also imatinib did not influence the cell viability in sphere-cultured SKOV3ip1 cells (Fig. 2A). Notably, BGJ398 differentially affected the cell viability in monolayer-cultured and sphere-cultured SKOV3ip1 (Fig. 2A and B). BGJ398 reduced the cell viability of sphere-cultured SKOV3ip1 cells in a dose-dependent manner (P>0.05; Fig. 2A). MTS assay was used to confirm viability of cells treated with inhibitors. The inhibition of cell viability by BGJ398 was also observed in sphere cultured SKOV3ip1 cells (P>0.05; Fig. 2B). BGJ398-treated sphere-cultured SKOV3ip1 cells did not maintain the healthy aggregate formation (Fig. 2D). Dissociation of spheroids were not observed 

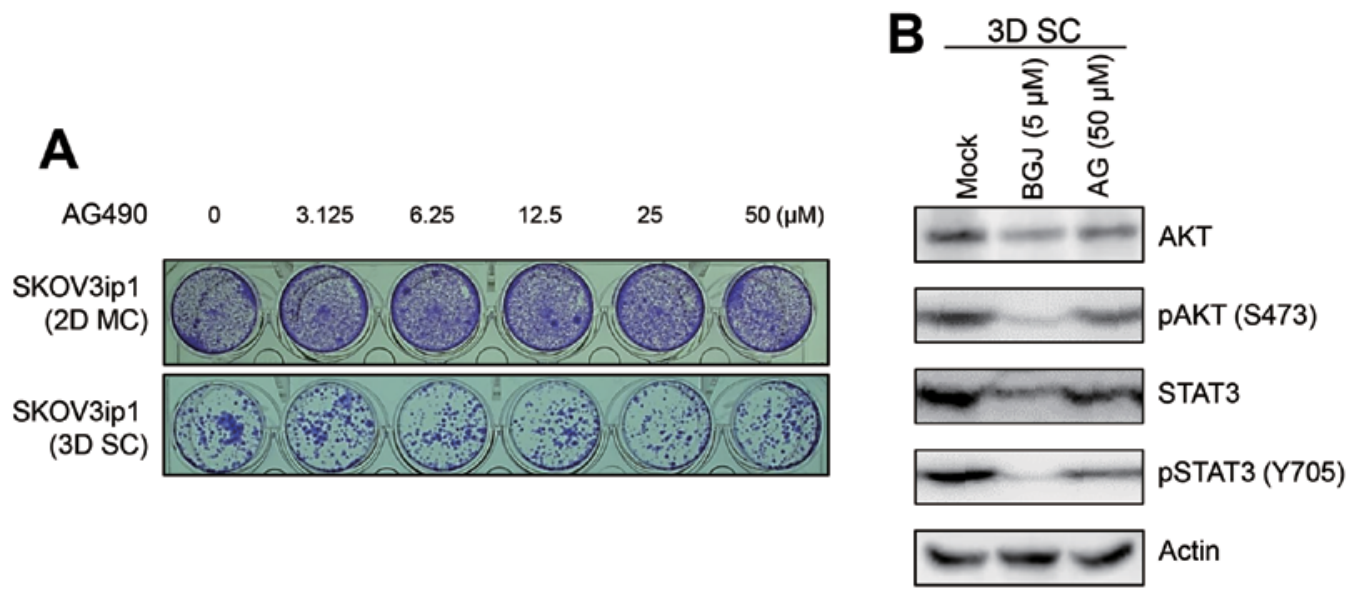

Figure 4. JAK inhibitor AG490 did not inhibit cell viability, nor downregulated the phosphorylation status of AKT and STAT3 in sphere-cultured SKOV3ip1 cells. (A) Cell viability of sphere-cultured SKOV3ip1 at $72 \mathrm{~h}$ after treatment with AG490 at the indicated concentration was analyzed by crystal violet staining. (B) Sphere-cultured SKOV3ip1 cells were treated with $5 \mu \mathrm{M}$ BGJ398 or AG490 $50 \mu \mathrm{M}$ AG490 for $72 \mathrm{~h}$. The expression and its phosphorylation of AKT and STAT3 were assessed by immunoblotting. Actin was used as a loading control.
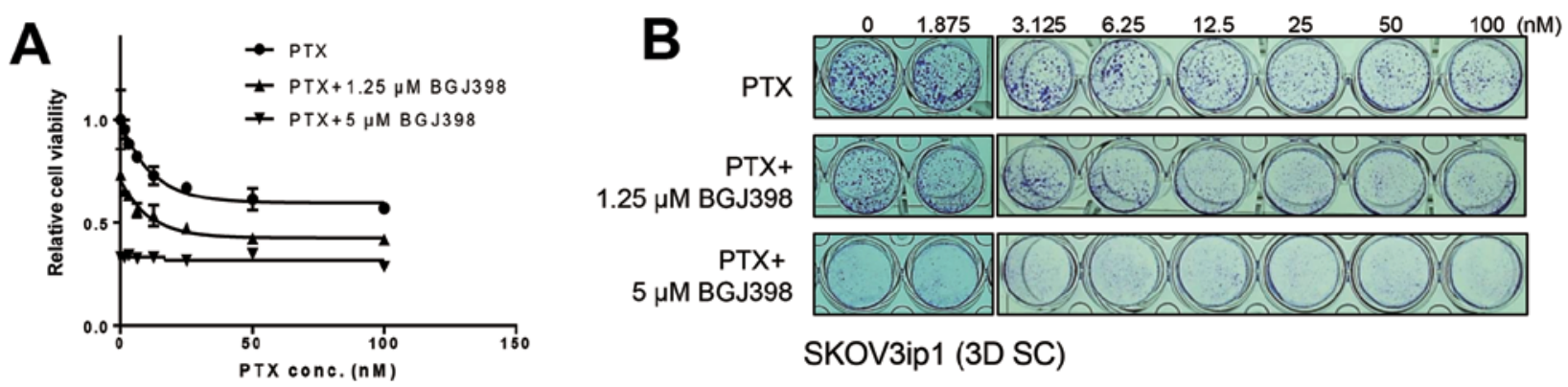

Figure 5. BJG398 enhances the cell viability inhibition of sphere cultured SKOV3ip1 with the combination treatment of paclitaxel. (A) Cell viability of sphere-cultured SKOV3ip1 at $72 \mathrm{~h}$ after treatment with serial diluted paclitaxel $(0,1.875,3.125,6.25,12.5,25,50$ and $100 \mathrm{nM})$ together with dose-different combinations $(0,1.25$ and $5 \mu \mathrm{M})$ of BGJ398 was determined by crystal violet colorimetric analysis. (B) Viable cells at $72 \mathrm{~h}$ after combination treatment of paclitaxel and BGJ398 at indicated concentration were visualized by crystal violet staining. All experiments were repeated three times. Significant differences by concentration were calculated using one-way ANOVA test and $\mathrm{P} \leq 0.05$ analyzed by concentration were considered significant.

at TAE684-treated and imatinib-treated sphere-cultured SKOV3ip1 (Fig. 2D). Next, we examined the cell viability assay with BGJ398 at 24-72 h. The result showed that the cell viability was decreased in a dose-dependent manner both in monolayer cultured model and sphere cultured model at 24-72 h (Fig. 2E). Interestingly, decreased cell viability was not significant in monolayer-cultured SKOV3ip1 cell at 48- and 72-h treatments ( $\mathrm{P}>0.05)$ (Fig. 2E), but in spherecultured SKOV3ip1 cells, the cell viability was gradually decreased in a time-dependent manner (Fig. 2E). These data revealed that BGJ398 is a potent chemotherapeutic agent against SKOV3p1 cells depending on sphere growth conditions.

BGJ398 inhibits the activated AKT and STAT3 in spherecultured SKOV3ip1 cells. Since major survival signaling molecules, AKT and STAT3 were activated in spherecultured SKOV3ip1 cells, we examined whether BGJ398 was able to affect the activation status of AKT and STAT3 in sphere-cultured SKOV3ip1 cells. Immunoblotting analysis indicated that BGJ398 suppressed the phosphorylated AKT at Ser473 residue and STAT3 at Tyr 705 residue in spherecultured SKOV3ip1 cells (Fig. 3A). Also BGJ398 decreased the phosphorylation of AKT at Ser473 residue and STAT3 at Tyr 705 residue in a dose-dependent manner (Fig. 3B). In contrast, TAE684 and imatinib did not affect the status of phosphorylation of AKT at Ser473 residue and STAT3 at Tyr 705 residue in sphere-cultured SKOV3ip1 cells (Fig. 3C). These results demonstrated that BGJ398 suppressed the activated AKT and STAT3 signaling pathway in sphere-cultured SKOV3ip1 cells, not in monolayer-cultured SKOV3ip1 cells. Several published studies have demonstrated that JAK/STAT signaling regulates PI3K/AKT pathway in mammalian cells $(35,36)$. Inhibition of the cell viability of sphere-cultured SKOV3ip1 cells by BGJ398 resulted in inhibition of AKT and STAT3 activation leading us to further examine whether JAK/STAT3 signaling mediates sphere culture-induced AKT and STAT3 activation in SKOV3ip1 cells. Thus, we examined whether the selective JAK tyrosine kinase inhibitor AG490 affected the activation of AKT and STAT3 status in spherecultured SKOV3ip1 cells. As shown in Fig. 4A, the treatment of AG490 is not able to inhibit cell growth of sphere-cultured SKOV3ip1 cells. Also, the phosphorylation of AKT and STAT3 was not inhibited by treatment of AG490 in spherecultured SKOV3ip1 cells (Fig. 4B). Collectively, these results indicated that BGJ398 effectively suppressed cell growth of 
sphere-cultured SKOV3ip1 through the inhibition of major survival signaling molecules, AKT and STAT3 activation irrelevant to JAK/STAT3 signaling pathway.

BGJ398 synergistically inhibits the cell viability of spherecultured SKOV3ipl with the combinational treatment of paclitaxel. Previous results that the metastatic microenvironment mimetic sphere culture of SKOV3ip1 enhances paclitaxel resistance led us test whether BGJ398 sensitizes paclitaxelresistance in sphere-cultured SKOV3ip1 cells. To determine whether the combination of BGJ398 and paclitaxel can synergistically inhibit cell viability in sphere-cultured SKOV3ip1, serial diluted paclitaxel with dose-different combinations $(0,1.25$ and $5 \mu \mathrm{M})$ of BGJ398 were treated in sphere-cultured SKOV3ip1 and crystal violet colorimetric analysis was examined at $72 \mathrm{~h}$ after treatment. As shown in Fig. 5, treatment of sphere-cultured SKOV3ip1 with BGJ398 in combination with paclitaxel led to a synergistic inhibition of cell viability compared to treatment of paclitaxel alone.

\section{Discussion}

Ovarian cancer is the most lethal among gynecological cancers (1). Despite the active pharmaceutical research against ovarian cancer to date, paclitaxel is still a first-line therapeutic anticancer drug for ovarian cancer (2). Moreover, the recurrent ovarian cancer in patients due to the development of chemoresistant phenotype is the main obstacle to managing advanced ovarian cancer (37). Together with understanding of the chemoresistance molecular mechanism of ovarian cancer, developing new pharmaceutical agents will be important to overcoming of ovarian cancer. We describe here a novel antitumor activity of pan-FGFR inhibitor BGJ398 against ovarian cancer dependent on sphere culture model. Also, BGJ398 induced dissociation of the spheroid formation of ovarian cancer cells.

Although many anticancer drugs showed promising effects in vitro cell line model, most failed to develop into an in vivo model system. Application of the modified in vitro culture model considering in vivo tumor microenvironment will be important at the developmental stage of anticancer drug. It has been reported sphere culture of epithelial ovarian carcinoma cells represented the feature of in vivo histological differentiation rather than the cells which were cultured in conventional monolayer model (38). Moreover, the transition from monolayer culture model to sphere culture model induced changes in the expression of molecular biomarkers relevant to malignancy including cadherins, vimentin and $\beta$-catenin (38). In this study, we suggested that the activated AKT and STAT3, the key regulators in cell survival, also changed by the transition of the culture model (Fig. 1) result in sensitizing the antitumoral activity of BGJ398 against SKOV3ip1. Many studies have reported the characterization of cancer cell spheroid as a model of cancer stem cells. Ovarian cancer cell spheroids with stem cell-like phenotype have been also reported to upregulate stem cell markers, metastatic capability and acquiring chemotherapy resistance (39-41). In the present study, we did not incubate SKOV3ip1 cells with stem cell enrichment media, nor separated the side population by FACS sorting for the enrichment of ovarian cancer stem cells. AKT and STAT3 activation was demonstrated by SKOV3ip1 incubation with anchorage-independent growth. Moon and colleagues (42) have reported that PI3K/Akt and Stat3 signaling are important roles in maintenance of cancer stem cells of glioblastoma cell model. Moreover, it has been reported that STAT3 signaling pathway has a role in drug-resistance, migration, and invasion and the blockade of STAT3 potentiates chemotherapy in ovarian cancer stem cell model (43-46). Although FGFR signaling function in maintenance of cancer stem cells has been reported (47), further examinations how ovarian cancer sphere culture system upregulates cellular AKT and STAT3 activation and what the molecular mechanism of FGFR inhibitor BGJ398 in regulation of AKT and STAT3 in sphere cultured-SKOV3ip1 were needed.

BGJ398 is a selective pan-FGFR inhibitor with $\mathrm{IC}_{50}$ of 0.9 , 1.4, 1.0 and $60 \mathrm{nM}$ for FGFR1, 2, 3 and 4 in cell-free assay, respectively (48) and with potential antiangiogenic and antineoplastic activities (11). Cheng et al (49) have reported that BGJ398 inhibited the growth of E-cadherin-positive epithelial type bladder cell lines at drug concentrations of $1 \mu \mathrm{M}$ or lower. They also have shown that BGJ-398 did not inhibit mesenchymal type bladder cancer cells and primary tumor growth but block tumor metastasis in a mouse model in vivo (49). In this study, we did not observe changes of signaling molecules on EMT in SKOV3ip1 cells following sphere cultivation. Although it has been reported that SKOV3ip1 is highly malignant and has metastasis potential by overexpressing ERBB2 (50), whether FGFR may be responsible for mediating EMT signaling in ovarian cancer spheres will be an important research area. TAE684 is a selective ALK kinase inhibitor (51). TAE684 treatment induces inhibition of phosphorylation of NPM-ALK and its downstream effectors including STAT3 and STAT5 and blocked the growth of anaplastic large-cell lymphoma (ALCL)-drived and ALK-dependent cell lines (51). Imatinib is a multi-target kinase inhibitor against Abl (52), PDGF (53), and c-Kit (54) used in the treatment of various cancers, mostly BCR/ABL-mutated chronic myelogenous leukemia. It has been reported that imatinib reverses doxorubicin-resistance by repression of the NF- $\mathrm{kB}$ signaling and preventing activation of STAT3/HSP27/p38/Akt survival pathway in highly active c-Abl cancer cells (55). Despite TAE684 having cytotoxic activity in monolayer-cultured SKOV3ipl cells as well as in spherecultured SKOV3ip1 cells, our data showed that TAE684 and imatinib do not suppress AKT and STAT3 pathways (Fig. 3A).

The present study showing the inhibition of the SKOV3ip1 ovarian cancer growth in sphere culture system by BGJ398 treatment implies that FGFR signaling potentiated the specific antitumor target against ovarian cancer spheroids. It has been reported that RNAi-induced FGFR1/2 reduction inhibits proliferation of SKOV3 ovarian cancer cell lines in vitro and increases cisplatin sensitivity (56). However, we demonstrated that significant cell toxicity was not observed in BGJ398treated monolayer-cultured SKOV3ip1 cells. Specifically, the cell viability was decreased by BGJ398 in sphere-cultured SKOV3ip1 cells (Fig. 2). Despite the absence of the expression profile of FGFR isotypes in sphere-cultured SKOV3ip cells, we revealed the downregulation of AKT and STAT3 activation via a pan-FGFR inhibition in sphere-cultured SKOV3ip1 cells (Fig. 3A). Furthermore, we observed cytotoxicity and dissociation of spheroids of SKOV3ip1 by treatment of 
PI3K/AKT inhibitor wortmannin in SKOV3ip1 cells implying that AKT activation is critical for the cell survival in spherecultured SKOV3ip1 cells (data not shown). Potent therapeutic targeting of the JAK/STAT3 pathway for ovarian cancer growth inhibition has been demonstrated $(57,58)$. Several reports have demonstrated that JAK signaling regulates PI3K/AKT pathway in cells $(35,36)$. Recently, Wen et al (59) reported that JAK/STAT3 inhibition using small molecule inhibitor suppressed tumor progression and metastasis in peritoneal mouse model of ovarian cancer. Moreover, it has been reported that the inhibition of Jak2 suppresses the progression of ovarian cancer (60). In the present study, we did not investigate the defined signaling mechanism of AKT and STAT3 activation in sphere-cultured SKOV3ip1 cells. The underlying molecular mechanisms remain to be elucidated.

As shown in Fig. 2, evidence have been reported that cells formed 3D spheroids have resistance to antitumor chemotherapeutics including paclitaxel and cisplatin. This has been largely considered due to several mechanisms including a decreased penetration rate of drugs into spheroid structure and alteration of prosurvival signaling pathway $(61,62)$. Cumulative evidence have showed that $\beta$-integrin signaling is important in the maintenance of ovarian cancer spheroid. Casey et al (63) reported that ovarian cancer spheroid formation and the adhesion of spheroids to extracellular matrix components is a $\beta$-integrin-dependent event. Sodek et al (64) demonstrated that the overexpression of $\beta 1$-integrin induces compact spheroid formation and invasive behavior in ovarian cancer cells. Yoshida et al (62) reported that laminin-1-derived synthetic peptide AG73 enhances the expression of $\beta 1$-integrin and induced the activation of downstream effector genes including MAPK, ERK and AKT. Therefore, we cannot rule out the possibility that the interference of the $\beta$-integrin pathway by BGJ398 may exist and contribute to BGJ398-induced cytotoxicity in sphere-cultured SKOV3ipl cells.

Solid tumors on primary tissue were organized upon its specific tumor microenvironment with cell-to-cell or cell-toextracellular matrix association. Unlike other cancers, ovarian cancer metastasis is distinctly developed by dissemination into peritoneal cavity and associated with the ascitic fluid to form metastatic microenvironment (65). Within ascitic fluid, ovarian cancer cells exist as individual cells or multicellular aggregates with absence of anchorage-dependent signaling. In ovarian cancer-specific microenvironment, metastases aggregates have an enhanced resistance to anticancer drugs, including paclitaxel (66). In the present study, BGJ398 sensitized the cell cytotoxic activity with a combination treatment of paclitaxel in sphere-cultured SKOV3ip1 cells implying a possibility of new therapeutic approaches for targeting against ovarian cancers spheroid regarding ovarian tumor microenvironment (Fig. 5).

In conclusion, the present study suggests that pan-FGFR inhibitor BGJ398 is a potent chemotherapeutic agent for ovarian cancer spheroid. Our data also indicated that FGFR may have a unique prosurvival role in the spheroid maintenance of ovarian cancer. This study gives us a more comprehensive insight into 3D sphere cell culture model during drug development considering ovarian cancer specific microenvironment and the antitumor activity of FGFR inhibitor against metastatic ovarian carcinoma.

\section{Acknowledgements}

The present study was supported by the Basic Science Research Program through the National Research Foundation of Korea (NRF) funded by the Ministry of Education (2015R1D1A1A01060688).

\section{References}

1. Jelovac D and Armstrong DK: Recent progress in the diagnosis and treatment of ovarian cancer. CA Cancer J Clin 61: 183-203, 2011.

2. Kampan NC, Madondo MT, McNally OM, Quinn M and Plebanski M: Paclitaxel and its evolving role in the management of ovarian cancer. BioMed Res Int 2015: 413076, 2015.

3. Vaughan S, Coward JI, Bast RC Jr, Berchuck A, Berek JS, Brenton JD, Coukos G, Crum CC, Drapkin R, Etemadmoghadam D, et al: Rethinking ovarian cancer: Recommendations for improving outcomes. Nat Rev Cancer 11: 719-725, 2011.

4. Brouwer-Visser J, Lee J, McCullagh K, Cossio MJ, Wang Y and Huang GS: Insulin-like growth factor 2 silencing restores taxol sensitivity in drug resistant ovarian cancer. PLoS One 9: e100165, 2014.

5. Kelleher FC, O'Sullivan H, Smyth E, McDermott R and Viterbo A: Fibroblast growth factor receptors, developmental corruption and malignant disease. Carcinogenesis 34: 2198-2205, 2013.

6. Hughes SE: Differential expression of the fibroblast growth factor receptor (FGFR) multigene family in normal human adult tissues. J Histochem Cytochem 45: 1005-1019, 1997.

7. Turner $\mathrm{N}$ and Grose R: Fibroblast growth factor signalling: From development to cancer. Nat Rev Cancer 10: 116-129, 2010.

8. Touat M, Ileana E, Postel-Vinay S, André F and Soria JC: Targeting FGFR signaling in cancer. Clin Cancer Res 21: 2684-2694, 2015.

9. Wesche J, Haglund K and Haugsten EM: Fibroblast growth factors and their receptors in cancer. Biochem J 437: 199-213, 2011.

10. Hallinan N, Finn S, Cuffe S, Rafee S, O'Byrne K and Gately K: Targeting the fibroblast growth factor receptor family in cancer. Cancer Treat Rev 46: 51-62, 2016.

11. Katoh M: FGFR inhibitors: Effects on cancer cells, tumor microenvironment and whole-body homeostasis (Review). Int J Mol Med 38: 3-15, 2016.

12. Helsten T, Elkin S, Arthur E, Tomson BN, Carter J and Kurzrock R: The FGFR landscape in cancer: Analysis of 4,853 tumors by next-generation sequencing. Clin Cancer Res 22: 259-267, 2016.

13. Xue M, Cao X, Zhong Y, Kuang D, Liu X, Zhao Z and Li H: Insulin-like growth factor-1 receptor (IGF-1R) kinase inhibitors in cancer therapy: Advances and perspectives. Curr Pharm Des 18: 2901-2913, 2012.

14. Asati V, Mahapatra DK and Bharti SK: PI3K/Akt/mTOR and Ras/Raf/MEK/ERK signaling pathways inhibitors as anticancer agents: Structural and pharmacological perspectives. Eur J Med Chem 109: 314-341, 2016.

15. Chen J, Elfiky A, Han M, Chen C and Saif MW: The role of Src in colon cancer and its therapeutic implications. Clin Colorectal Cancer 13: 5-13, 2014.

16. Schultze SM, Hemmings BA, Niessen M and Tschopp O: PI3K/ AKT, MAPK and AMPK signalling: Protein kinases in glucose homeostasis. Expert Rev Mol Med 14: e1, 2012.

17. Dillon LM and Miller TW: Therapeutic targeting of cancers with loss of PTEN function. Curr Drug Targets 15: 65-79, 2014.

18. Franke TF, Yang SI, Chan TO, Datta K, Kazlauskas A, Morrison DK, Kaplan DR and Tsichlis PN: The protein kinase encoded by the Akt proto-oncogene is a target of the PDGFactivated phosphatidylinositol 3-kinase. Cell 81: 727-736, 1995.

19. Wu J, Feng X, Zhang B, Li J, Xu X, Liu J, Wang X, Wang J and Tong X: Blocking the bFGF/STAT3 interaction through specific signaling pathways induces apoptosis in glioblastoma cells. J Neurooncol 120: 33-41, 2014. 
20. Kohn AD, Kovacina KS and Roth RA: Insulin stimulates the kinase activity of RAC-PK, a pleckstrin homology domain containing ser/thr kinase. EMBO J 14: 4288-4295, 1995.

21. Henson ES and Gibson SB: Surviving cell death through epidermal growth factor (EGF) signal transduction pathways: Implications for cancer therapy. Cell Signal 18: 2089-2097, 2006.

22. Hemmings BA and Restuccia DF: PI3K-PKB/Akt pathway. Cold Spring Harb Perspect Biol 4: a011189, 2012.

23. Altomare DA and Testa JR: Perturbations of the AKT signaling pathway in human cancer. Oncogene 24: 7455-7464, 2005.

24. Kim D, Dan HC, Park S, Yang L, Liu Q, Kaneko S, Ning J, He L, Yang $\mathrm{H}$, Sun M, et al: AKT/PKB signaling mechanisms in cancer and chemoresistance. Front Biosci 10: 975-987, 2005.

25. Mehta G, Hsiao AY, Ingram M, Luker GD and Takayama S: Opportunities and challenges for use of tumor spheroids as models to test drug delivery and efficacy. J Control Release 164 192-204, 2012.

26. Hickman JA, Graeser R, de Hoogt R, Vidic S, Brito C, Gutekunst $\mathrm{M}$ and van der Kuip H; IMI PREDECT Consortium: Three-dimensional models of cancer for pharmacology and cancer cell biology: Capturing tumor complexity in vitro/ex vivo. Biotechnol J 9: 1115-1128, 2014.

27. Hirschhaeuser F, Menne H, Dittfeld C, West J, Mueller-Klieser W and Kunz-Schughart LA: Multicellular tumor spheroids: An underestimated tool is catching up again. J Biotechnol 148: 3-15, 2010.

28. Ke N, Albers A, Claassen G, Yu DH, Chatterton JE, Hu X, Meyhack B, Wong-Staal F and Li QX: One-week 96-well soft agar growth assay for cancer target validation. Biotechniques 36 : 826-828, 830, 832-823, 2004.

29. Cao D, Kishida S, Huang P, Mu P, Tsubota S, Mizuno M and Kadomatsu K: A new tumorsphere culture condition restores potentials of self-renewal and metastasis of primary neuroblastoma in a mouse neuroblastoma model. PLoS One 9: e86813, 2014.

30. Weiswald LB, Bellet D and Dangles-Marie V: Spherical cancer models in tumor biology. Neoplasia 17: 1-15, 2015 .

31. Ahmed N and Stenvers KL: Getting to know ovarian cancer ascites: Opportunities for targeted therapy-based translational research. Front Oncol 3: 256, 2013.

32. Yu D, Wolf JK, Scanlon M, Price JE and Hung MC: Enhanced c-erbB-2/neu expression in human ovarian cancer cells correlates with more severe malignancy that can be suppressed by E1A. Cancer Res 53: 891-898, 1993 .

33. Dieci MV, Arnedos M, Andre F and Soria JC: Fibroblast growth factor receptor inhibitors as a cancer treatment: From a biologic rationale to medical perspectives. Cancer Discov 3: 264-279, 2013.

34. Ornitz DM and Itoh $\mathrm{N}$ : The fibroblast growth factor signaling pathway. Wiley Interdiscip Rev Dev Biol 4: 215-266, 2015.

35. Yamada O, Ozaki K, Akiyama M and Kawauchi K: JAK-STAT and JAK-PI3K-mTORC1 pathways regulate telomerase transcriptionally and posttranslationally in ATL cells. Mol Cancer Ther 11: 1112-1121, 2012.

36. Gu YJ, Sun WY, Zhang S, Li XR and Wei W: Targeted blockade of JAK/STAT3 signaling inhibits proliferation, migration and collagen production as well as inducing the apoptosis of hepatic stellate cells. Int J Mol Med 38: 903-911, 2016.

37. Jayson GC, Kohn EC, Kitchener HC and Ledermann JA: Ovarian cancer. Lancet 384: 1376-1388, 2014.

38. Lee JM, Mhawech-Fauceglia P, Lee N, Parsanian LC, Lin YG, Gayther SA and Lawrenson K: A three-dimensional microenvironment alters protein expression and chemosensitivity of epithelial ovarian cancer cells in vitro. Lab Invest 93: 528-542, 2013.

39. Liao J, Qian F, Tchabo N, Mhawech-Fauceglia P, Beck A, Qian Z, Wang X, Huss WJ, Lele SB, Morrison CD, et al: Ovarian cancer spheroid cells with stem cell-like properties contribute to tumor generation, metastasis and chemotherapy resistance through hypoxia-resistant metabolism. PLoS One 9: e84941, 2014.

40. He QZ, Luo XZ, Wang K, Zhou Q, Ao H, Yang Y, Li SX, Li Y, Zhu HT and Duan T: Isolation and characterization of cancer stem cells from high-grade serous ovarian carcinomas. Cell Physiol Biochem 33: 173-184, 2014.

41. Wang L, Mezencev R, Bowen NJ, Matyunina LV and McDonald JF: Isolation and characterization of stem-like cells from a human ovarian cancer cell line. Mol Cell Biochem 363: $257-268,2012$
42. Moon SH, Kim DK, Cha Y, Jeon I, Song J and Park KS: PI3K/ Akt and Stat 3 signaling regulated by PTEN control of the cancer stem cell population, proliferation and senescence in a glioblastoma cell line. Int J Oncol 42: 921-928, 2013.

43. Han Z, Feng J, Hong Z, Chen L, Li W, Liao S, Wang X, Ji T, Wang S, Ma D, et al: Silencing of the STAT3 signaling pathway reverses the inherent and induced chemoresistance of human ovarian cancer cells. Biochem Biophys Res Commun 435 188-194, 2013.

44. Han Z, Hong Z, Gao Q, Chen C, Hao Z, Ji T, Hu W, Yan Y, Feng J, Liao S, et al: A potent oncolytic adenovirus selectively blocks the STAT3 signaling pathway and potentiates cisplatin antitumor activity in ovarian cancer. Hum Gene Ther 23: 32-45, 2012.

45. Zhang X, Liu P, Zhang B, Wang A and Yang M: Role of STAT3 decoy oligodeoxynucleotides on cell invasion and chemosensitivity in human epithelial ovarian cancer cells. Cancer Genet Cytogenet 197: 46-53, 2010.

46. Fujiwara Y, Takaishi K, Nakao J, Ikeda T, Katabuchi H, Takeya M and Komohara Y: Corosolic acid enhances the antitumor effects of chemotherapy on epithelial ovarian cancer by inhibiting signal transducer and activator of transcription 3 signaling. Oncol Lett 6: 1619-1623, 2013

47. Chang JY, Wang C, Liu J, Huang Y, Jin C, Yang C, Hai B, Liu F, D'Souza RN, McKeehan WL, et al: Fibroblast growth factor signaling is essential for self-renewal of dental epithelial stem cells. J Biol Chem 288: 28952-28961, 2013.

48. Guagnano V, Kauffmann A, Wöhrle S, Stamm C, Ito M, Barys L, Pornon A, Yao Y, Li F, Zhang Y, et al: FGFR genetic alterations predict for sensitivity to NVP-BGJ398, a selective pan-FGFR inhibitor. Cancer Discov 2: 1118-1133, 2012.

49. Cheng T, Roth B, Choi W, Black PC, Dinney C and McConkey DJ: Fibroblast growth factor receptors-1 and -3 play distinct roles in the regulation of bladder cancer growth and metastasis: Implications for therapeutic targeting. PLoS One 8: e57284, 2013.

50. Ueno NT, Bartholomeusz C, Herrmann JL, Estrov Z, Shao R, Andreeff M, Price J, Paul RW, Anklesaria P, Yu D, et al: E1A-mediated paclitaxel sensitization in HER-2/neu-overexpressing ovarian cancer SKOV3.ip1 through apoptosis involving the caspase-3 pathway. Clin Cancer Res 6: 250-259, 2000.

51. Galkin AV, Melnick JS, Kim S, Hood TL, Li N, Li L, Xia G, Steensma R, Chopiuk G, Jiang J, et al: Identification of NVP-TAE684, a potent, selective, and efficacious inhibitor of NPM-ALK. Proc Natl Acad Sci USA 104: 270-275, 2007.

52. Buchdunger E, Zimmermann J, Mett H, Meyer T, Müller M, Druker BJ and Lydon NB: Inhibition of the Abl protein-tyrosine kinase in vitro and in vivo by a 2-phenylaminopyrimidine derivative. Cancer Res 56: 100-104, 1996.

53. Ranza E, Mazzini G, Facoetti A and Nano R: In-vitro effects of the tyrosine kinase inhibitor imatinib on glioblastoma cell proliferation. J Neurooncol 96: 349-357, 2010.

54. Heinrich MC, Griffith DJ, Druker BJ, Wait CL, Ott KA and Zigler AJ: Inhibition of c-kit receptor tyrosine kinase activity by STI 571, a selective tyrosine kinase inhibitor. Blood 96: 925-932, 2000.

55. Sims JT, Ganguly SS, Bennett H, Friend JW, Tepe J and Plattner R: Imatinib reverses doxorubicin resistance by affecting activation of STAT3-dependent NF- $\kappa$ B and HSP27/ p38/AKT pathways and by inhibiting ABCB1. PLoS One 8: e55509, 2013.

56. Cole C, Lau S, Backen A, Clamp A, Rushton G, Dive C, Hodgkinson C, McVey R, Kitchener H and Jayson GC: Inhibition of FGFR2 and FGFR1 increases cisplatin sensitivity in ovarian cancer. Cancer Biol Ther 10: 495-504, 2010.

57. Gritsina G, Xiao F, O'Brien SW, Gabbasov R, Maglaty MA, Xu RH, Thapa RJ, Zhou Y, Nicolas E, Litwin S, et al: Targeted blockade of JAK/STAT3 signaling inhibits ovarian carcinoma growth. Mol Cancer Ther 14: 1035-1047, 2015.

58. McCann GA, Naidu S, Rath KS, Bid HK, Tierney BJ, Suarez A, Varadharaj S, Zhang J, Hideg K, Houghton P, et al: Targeting constitutively-activated STAT3 in hypoxic ovarian cancer, using a novel STAT3 inhibitor. Oncoscience 1: 216-228, 2014.

59. Wen W, Liang W, Wu J, Kowolik CM, Buettner R, Scuto A, Hsieh MY, Hong H, Brown CE, Forman SJ, et al: Targeting JAK1/STAT3 signaling suppresses tumor progression and metastasis in a peritoneal model of human ovarian cancer. Mol Cancer Ther 13: 3037-3048, 2014. 
60. Kobayashi A, Tanizaki Y, Kimura A, Ishida Y, Nosaka M, Toujima S, Kuninaka Y, Minami S, Ino K and Kondo T: AG490, a Jak2 inhibitor, suppressed the progression of murine ovarian cancer. Eur J Pharmacol 766: 63-75, 2015.

61. Desoize B and Jardillier J: Multicellular resistance: A paradigm for clinical resistance? Crit Rev Oncol Hematol 36: 193-207, 2000.

62. Yoshida Y, Kurokawa T, Nishikawa Y, Orisa M, Kleinman HK and Kotsuji F: Laminin-1-derived scrambled peptide AG73T disaggregates laminin-1-induced ovarian cancer cell spheroids and improves the efficacy of cisplatin. Int J Oncol 32: 673-681, 2008.

63. Casey RC, Burleson KM, Skubitz KM, Pambuccian SE, Oegema TR Jr, Ruff LE and Skubitz AP: Beta 1-integrins regulate the formation and adhesion of ovarian carcinoma multicellular spheroids. Am J Pathol 159: 2071-2080, 2001.
64. Sodek KL, Ringuette MJ and Brown TJ: Compact spheroid formation by ovarian cancer cells is associated with contractile behavior and an invasive phenotype. Int J Cancer 124: 2060-2070, 2009.

65. Lengyel E: Ovarian cancer development and metastasis. Am J Pathol 177: 1053-1064, 2010

66. Thibault B, Castells M, Delord JP and Couderc B: Ovarian cancer microenvironment: Implications for cancer dissemination and chemoresistance acquisition. Cancer Metastasis Rev 33: 17-39, 2014. 